\title{
Imagerie des glandes annexes de la voie séminale
}

\author{
J.F. LAPRAY 1 , J. ROLLET ${ }^{2}$, F. ROBERT ${ }^{2}$ \\ ${ }^{1}$ Centre de radiologie, 151, avenue de Saxe, 69003, Lyon \\ ${ }^{2}$ Institut Rhônalpin, 1, rue Laborde, 69500, Bron
}

\section{RESUME}

L'imagerie des glandes annexes de la voie séminale qui repose sur l'échographie est orientée vers la recherche des obstructions congénitales ou acquises et de leur cause. Le bilan initial que nous utilisons depuis 10 ans comporte une étude échographique de l'ensemble de l'appareil génito-urinaire (reins, scrotum, carrefour uro-génital par voie endorectale).

Nous rappelons brièvement la technique et les images normales obtenues en échographie et en IRM.

La pathologie épididymaire est dominée par le retentissement, parfois discret, des obstructions d'aval. L'échographie permet de visualiser les lésions mécaniques (en permettant éventuellement une orientation des prélèvements) et inflammatoires qui sont souvent associées.

L'échographie endorectale (EER) permet de confirmer ou d'affirmer l'absence (uni- ou bilatérale) des canaux déférents. La vésicule séminale (VS) apparaît comme un bon marqueur de la présence des terminaisons déférentielles. L'existence d'une agénésie rénale associée n'incite pas à la recherche d'une mutation de la mucoviscidose, mais oriente vers une anomalie de développement embryologique de type wolffien. L'IRM apparaît toujours l'examen de choix en cas d'insuffisance de l'EER.

Même en cas d'obstacle proximal avéré, l'EER est nécessaire pour affirmer l'absence d'obstacle distal avant la réalisation d'une anastomose chirurgicale.
En effet, L'EER (comme l'échographie scrotale sur le plan proximal) permet d'apprécier par la dilatation le retentissement d'un obstacle d'aval sur la voie séminale. Elle peut parfois le mettre en évidence : absence de canaux déférents, kystes prostatiques, infections et leurs séquelles, lithiases... Mais à côté de situations avec des anomalies manifestes pour lesquelles l'échographie est décisive, il existe de nombreux cas (oligoasthénospermies, pathologie mixte secrétoire et excrétoire, etc...) où elle n'est, après l'examen clinique, le bilan hormonal et le spermogramme de départ, qu'un des éléments d'orientation. Elle bénéficie alors de la corrélation avec les marqueurs, et constitue un des éléments qui vont construire la décision.

Mots Clés : infertilité masculine, imagerie, échographie, IRM, voie séminale

\section{INTRODUCTION}

L'imagerie de la voie séminale était réservée avant le développement de l'échographie à la déférentographie dont les risques potentiels et les aléas de réalisation sont connus, mais qui reste la seule méthode capable d'affirmer la perméabilité de la voie excrétrice du sperme. Les glandes annexes (canal épididymaire, canal déférent, vésicules séminales, prostate) sont étagées sur la voie séminale qui devient la voie génito-urinaire à partir de l'urètre. 
L'échographie apparaît indiquée dans tout bilan d'une hypofertilité masculine dont l'origine excrétoire est possible. Ce bilan échographique d'une infertilité masculine comporte systématiquement dans notre expérience depuis 10 ans l'étude des reins, du contenu scrotal et du confluent vésiculo-déférentiel par par voie endorectale (EER). L'EER est parfois le prélude d'autres explorations d'imagerie, en particulier par IRM, la méthode de guidage de ponctions - avec éventuelles opacifications radiologiques - des vésicules séminales ou des kystes de la base prostatique, ou un élément de contrôle peropératoire lors d'une résection endoscopique trans-urétrale (RTU).

\section{TECHNIQUE ET RESULTATS NORMAUX}

\section{1. Échographie scrotale}

Elle est effectuée avec une sonde de haute fréquence $\geq 7,5 \mathrm{Mhz}$ permettant l'étude Dopplercouleur devenue un complément indispensable de l'examen [4].

L'épididyme apparaît homogène et sensiblement isoéchogène au testicule auquel il est accolé. Ses contours sont nets et réguliers. La tête, d'examen facile, est de forme grossièrement triangulaire avec des bords convexes, et une épaisseur voisine de $10 \mathrm{~mm}$ [3]. Le corps situé sur la face postérieure ou externe du testicule apparaît comme une structure rubannée d'environ 2 à $4 \mathrm{~mm}$ et se poursuit par la queue épididymaire, arciforme, et la jonction épididymo-déférentielle, qui sont d'examen plus difficile (fig.1). Le canal déférent, rarement étudié, est décrit comme une structure tubulaire d'environ $2 \mathrm{~mm}$ de diamètre au bord externe du cordon repérable par la mise en évidence des vaisseaux spermatiques au Doppler-couleur [17].

\section{2. Échographie endorectale}

Elle est réalisée en décubitus latéral, à vessie vide, avec une sonde (volontiers multiplans) de haute fréquence $\geq 7,5 \mathrm{Mhz}$. L'utilisation du Doppler-couleur peut faciliter l'examen. L'étude préalable avec une sonde de 3,5 Mhz, par voie sus-pubienne, à vessie pleine, de la paroi vésicale et d'une éventuelle masse supraprostatique est toujours utile d'autant qu'elle

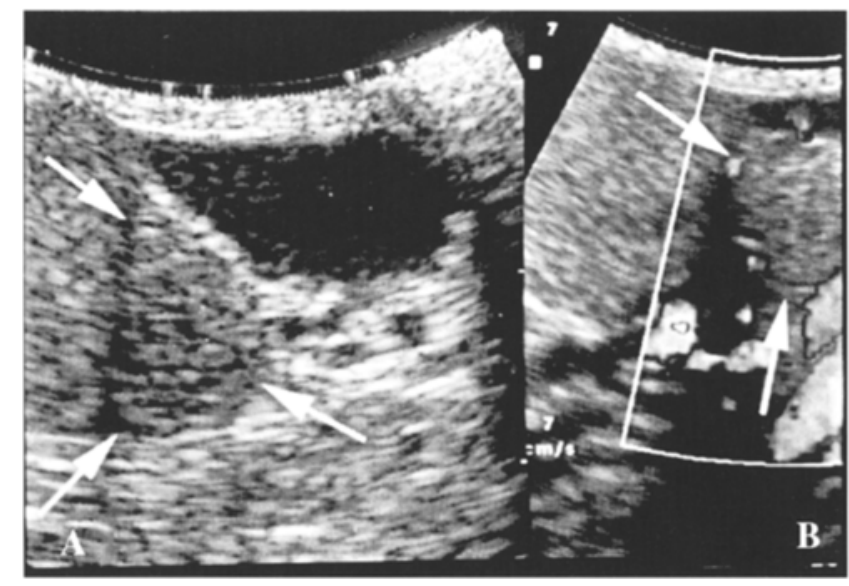

Figure 1 : échographie scrotale. a) queue épididymaire normale $(\rightarrow)$ au contact du pôle inférieur du testicule et $d$ 'une lame d'hydrocèle. b) l'utilisation du codage-couleur permet de différencier les structures vasculaires (varicocèle) des dilatations canalaires épididymaires.

complète l'examen des deux reins qui est indispensable.

L'EER permet l'étude des ampoules déférentielles (AD), et souvent des derniers centimétres préampullaires : présence, morphologie, symétrie, des vésicules séminales (VS) : morphologie, taille, contenu et parois glandulaires, ainsi que de la prostate : volumétrie, canaux éjaculateurs (CE), structures de la zone centrale et de la région sus-montanale.

Les $\mathrm{AD}$ apparaissent en coupe transversale comme deux structures arrondies hypoéchogènes situées dans le dièdre constitué par l'extrémité interne des VS, structures rubannées hypoéchogènes, de dimensions variables, avec parfois visibilité de logettes glandulaires liquidiennes (fig.2) [7]. La réunion des VS et des canaux déférents qui constitue le confluent vésiculo-déférentiel se produit dans $80 \%$ des cas immédiatement au dessus de la base prostatique [3].

Chez l'homme jeune de moins de 30 ans, la prostate est isoéchogène car la zone de transition n'est en général pas ou peu développée. Chez l'homme plus âgé, la zone de transition se différencie de la zone périphérique par un aspect plus hypoéchogène, parfois hétérogène et nodulaire. Les CE sont repérables dans la prostate centrale par leur fine paroi échogène, d'aspect punctiforme ou arrondi sur les coupes 


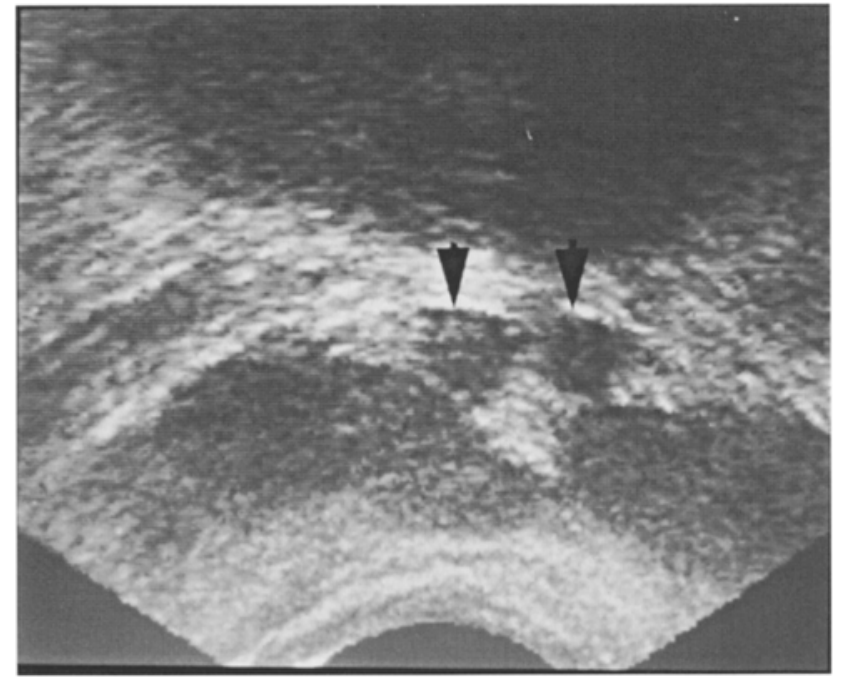

Figure 2 : échographie transrectale, coupe axiale. Les deux ampoules déférentielles arrondies (A) sont situées dans le dièdre formé par l'extrémité interne des deux vésicules séminales.

axiales, et sur les coupes sagittales comme une ligne unique ou dédoublée dans l'axe de l'urètre sous-montanal, en direction du veru montanum où ils se jettent dans l'urètre (fig.3) [23]. Le doppler-couleur peut faciliter leur repérage par la visibilité de l'artère qui longe leur paroi.

L'EER permet le prélévement de sperme dans les VS et la réalisation d'opacifications radiologiques de la distalité de la voie séminale pour la confirmation d'une obstruction en évitant une déférentographie $[8,10,18]$.

\section{IRM du confluent vésiculo-séminal}

Dans des cas sélectionnés, et en particulier en cas d'insuffisance de l'EER ou avant une exploration invasive ou une intervention chirurgicale, l'IRM apparaît comme la méthode d'investigation complémentaire la plus adaptée. Elle offre en effet une imagerie dans les 3 plans de l'espace ainsi qu'une analyse structurale que ne permet pas la tomodensitométrie qui n' a pratiquement pas d'indication dans cette pathologie $[3,13,15,21]$.

L'antenne endorectale permet en général une étude d'une excellente définition de la prostate, du confluent vésiculo-déférentiel (surtout séquences frontales) et des vésicules séminales

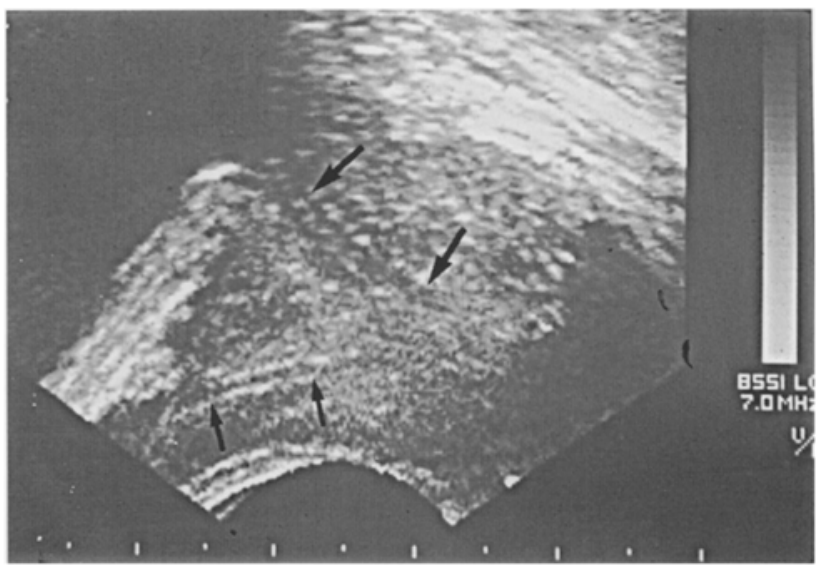

Figure 3 : échographie transrectale, coupe sagittale. Les parois d'un canal éjaculateur $(\rightarrow)$ sont visibles à la partie postérieure de la base prostatique en direction du veru montanum où se produit la réunion avec l'urètre susmontanale $(\rightarrow)$.

tandis que l' antenne corps est nécessaire pour l'examen des anomalies supra-prostatiques, en particulier des volumineuses lésions kystiques qui échappent au champ limité de l'antenne endorectale. Il en est de même lorsque des mensurations précises de l'épaisseur prostatique rétro-urétrale sans perturbation par une sonde ou une antenne endorectales sont souhaitables (séquences sagittales avant RTU d'une paroi d'un kyste au contact du veru).

L'examen utilise des séquences en fast spin écho (FSE) en T1 et en T2, avec des coupes de l'ordre de $4 \mathrm{~mm}$ d'épaisseur.

Les séquences en T1 où les VS et la prostate ont un signal homogène et intermédiaire sont surtout utiles dans cette pathologie pour analyser le contenu pur ou impur des structures liquidiennes.

En T2, les AD ont une paroi épaisse en hyposignal, centrée par l'hypersignal du contenu de la lumière. Les parois externes et internes des VS, fines, sont en hyposignal et le contenu glandulaire est en hypersignal homogène (fig.4). L'anatomie zonale de la prostate est visible avec la zone périphérique en hypersignal et la zone centrale en hyposignal, où les canaux éjaculateurs sont généralement repérables par l' hypersignal de leur contenu liquidien 


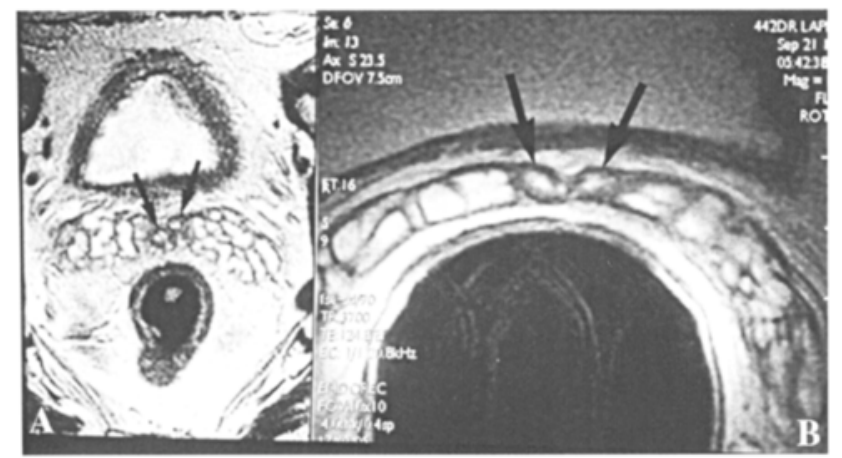

Figure 4 : IRM séquence axiale T2 avec antenne corps (a) et antenne endorectale (b). Les ampoules déférentielles $(\rightarrow)$ sont visibles en dedans des vésicules séminales.

\section{PATHOLOGIE}

La pathologie de l'hypofertilité excrétoire est dominée par les obstructions complètes ou incomplètes de la voie séminale et les lésions infectieuses ou inflammatoires du tractus génito-urinaire. Ces lésions mécaniques et infectieuses sont souvent associées, les unes pouvant être la cause des autres.

\section{L'épididyme}

L'existence d'un obstacle d'aval va typiquement se traduire par une hypertrophie des différentes portions de l'épididyme, avec la visibilité directe des dilatations canaliculaires (fig.5). Mais l'aspect peut être plus discret avec des plages hypoéchogènes, souvent nodulaires, visibles au niveau de la tête et de la queue de l'épididyme (fig.6). Ces images sont considérées comme des zones de dilatation canalaires comme le montrent les sondes à très haute fréquence $(>10 \mathrm{MHz})$. A l'inverse, la distension épididymaire peut être majeure avec la confirmation échographique de spermatocèles palpables apparaissant comme des nodules liquidiens parfois volumineux. La présence d'une varicocèle, fréquente du côté gauche, nécessite le recours systématique à l'étude Doppler-couleur pour différencier les dilatations canalaires épididymaires des dilatations veineuses.

Dans tous les cas il conviendra d'examiner le testicule, dont la pathologie variée est exclue de notre sujet, à la recherche de dilatation des tubes efférents ou de dilatations microkystiques du rete testis, témoignant du retentissement de l'obstruction d'aval (fig.7) [1].
Les séquelles inflammatoires et infectieuses vont au contraire se traduire par des zones hyperéchogènes mais les rétractions engendrées par ces lésions cicatricielles sont responsables de sténoses avec des dilatations d'amont ce qui fait que le tableau échographique est souvent mixte.

La présence de matériel échogène à l'intérieur des tubes épididymaires dilatés reste discutée, plutôt évocatrice d'un liquide de stase échogène [17].

L'interprétation de l'ensemble de ces images est parfois délicate.

L'absence de la queue épididymaire (et parfois du corps) évoque d'emblée une absence de canal déférent qui va être recherchée par EER et s'accompagne presque toujours de dilatations canalaires de l'épididyme restant. (fig.8)

\section{Les ampoules déférentielles}

La non visibilité des $2 \mathrm{AD}$ (sans agénésie rénale associée) suggère le diagnostic d'absence bilatérale des canaux déférents (ABCD) (anglais : $C B A V D$, congenital bilateral absence of the vas deferens). L'ABCD est responsable de 1 à $2 \%$ des cas de stérilité masculine et d'environ 6 à $25 \%$ des cas d'azoospermie d'origine obstructive. Elle est présente chez $98 \%$ des patients atteints de mucoviscidose, maladie héréditaire se transmettant sur le mode autosomique récessif, en rapport avec le gène codant pour la protéine CFTR (cystic fibrosis transmembrane conductance regulator). Des mutations dans le gène CFTR ont été constatées chez 41 à $64 \%$ des patients porteurs d'une $A B C D$, ce qui est nettement supérieur au portage hétérozygote dans la population générale qui est d'environ $4 \%$ [19]. L'ABCD isolée est donc actuellement considérée comme une forme mineure de l'expression phénotypique de la mucoviscidose. Parmi les multiples mutations décrites, la mutation $\Delta F 508$ est la plus fréquente.

Dans notre série rétrospective portant sur 463 hommes infertiles dont 109 patients azoospermiques ayant subi un bilan échographique entre janvier 1993 et mars 1998, nous observons $51 \mathrm{ABCD}$ dont 32 cas d'ABCD sans agénésie rénale, et 17 cas d'absence unilatérale d'un canal déférent (AUCD) dont 7 sans agénésie rénale associée (tableau 1) [19]. 


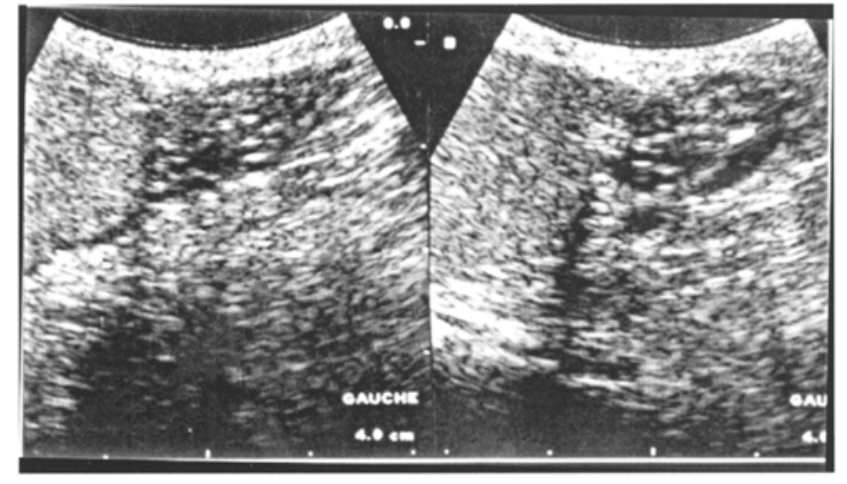

Figure 5 : échographie scrotale : multiples dilatations canalaires de la queue épididymaire témoignant d'un obstacle d'aval sur deux coupes sagittales $a$ et $b$.

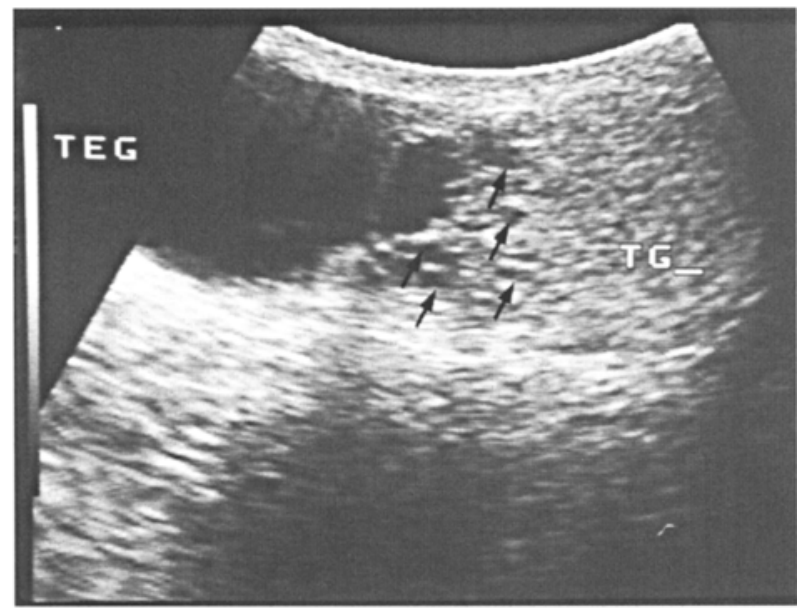

Figure 7 : nombreuses dilatations canalaires intratesticulaires $(\rightarrow)$ au contact d'un volumineux kyste (spermatocèle) de la tête épididymaire.

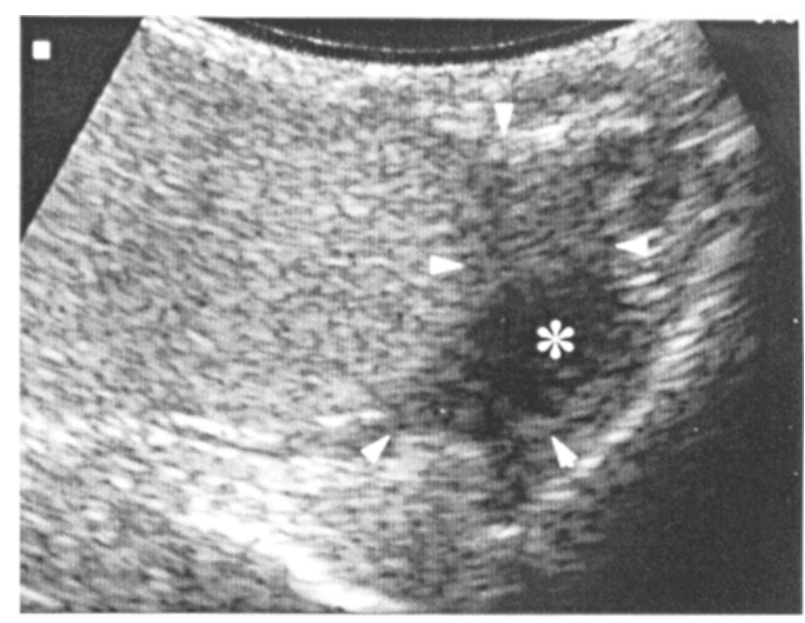

Figure 6 : échographie scrotale : plage hypoéchogène (*) témoignant de dilatations canaliculaires au sein de la queue épididymaire modérément hypertrophiée (A).

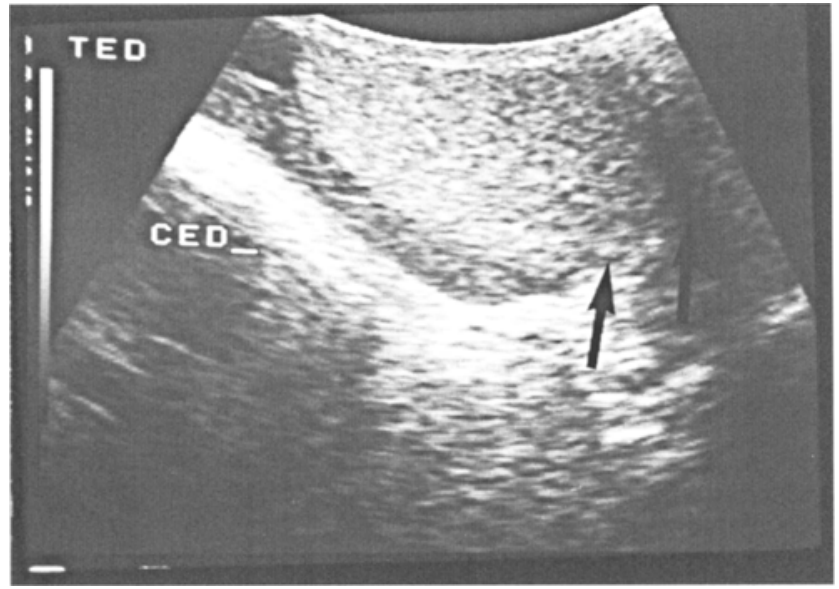

Figure 8 : échographie scrotale : absence de queue épididymaire $(\rightarrow)$ et multiples dilatations canalaires du corps et de la tête épididymaires.

Tableau 1 : répartition des azoospermies dans notre série échographique [19].

\begin{tabular}{lcc} 
& $\mathbf{1 0 9}$ azoospermies & $\mathbf{4 6 3}$ infertiles \\
\hline ABCD & $31,2 \%$ & $7,3 \%$ \\
AUCD & $5,5 \%$ & $3,7 \%$ \\
Obstacles épididymo- & & \\
déférentiels & $25,7 \%$ & - \\
kystes prostatiques & 0 & $2,8 \%$ \\
mixtes & $10,1 \%$ & - \\
insuffisance secrétoire & $27,5 \%$ & - \\
\hline
\end{tabular}


L'ABCD s'accompagne fréquemment de l'absence de l'une ou des deux VS (présence d'1 VS dans $53 \%$, de 2 VS dans $12,5 \%$, et absence des 2 VS dans $34 \%$ des cas de notre série).

Quand celles-ci restent présentes, de façon uniou bilatérale, elles sont presque toujours anormales (100\% dans notre série) (fig.9). L'aspect de coque liquidienne avec une déshabitation glandulaire est largement plus fréquent que celui d'une VS atrophique ou dystrophique avec des parois épaissies, une structure hyperéchogène, voire des calcifications.

Face à une azoospermie avec hypospermie, l'examen clinique expérimenté fait facilement le diagnostic par la palpation négative des déférents dans les bourses, associée à une distension épididymaire en amont de l'obstacle d'aval, d'autant que la queue et parfois le corps épididymaires, de même origine embryologique que le déférent, sont absents. Mais la présence d'un scrotum épais, d'anomalies associées ou de formes incomplètes avec présence proximale et absence distale du déférent qui sont importantes sur le plan pathogénique, ont fait de l'échographie un complément quasiindispensable de l'examen clinique.

En effet plusieurs constatations étayent l'hypothèse d'une involution des structures épididymo-vésiculo-déférentielles et non d'une agénésie vraie par absence de développement du

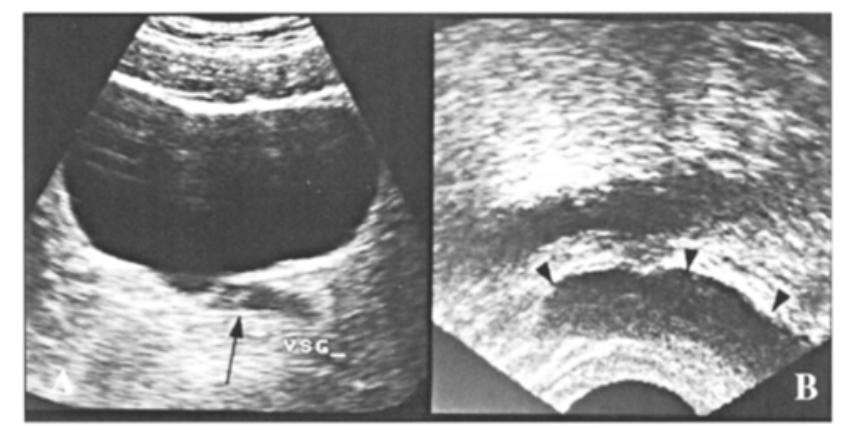

Figure 9 : absence bilatérale des canaux déférents : échographie, (a) par voie sus-pubienne, visibilité d'une seule vésicule séminale, du côté gauche $(\rightarrow)$. La coupe axiale par voie endorectale (b) confirme l'absenced'ampoules déférentielles ou de vésicule séminale droite. La vésicule séminale gauche unique (A) apparaît comme une coque liquidienne sans structure glandulaire. canal de Wolff, ce qui incite à préférer le terme d'absence de canaux déférents à celui d'agénésie dans cette pathologie [20].

L'EER permet d'affirmer le diagnostic d'ABCD. En dehors de difficultés techniques liées à la morphologie du patient, la limite nous paraît être les cas où une volumineuse VS distendue occupant la partie centrale de l'espace susprostatique ne permet plus d'affirmer formellement l'absence d' ampoule déférentielle ( 3 cas dans notre série), ce que pourrait résoudre une IRM du carrefour. L'échographie permet bien sûr l'affirmation de la présence des 2 reins, ainsi que l'analyse épididymaire (fig.8).

Il n' existe pas dans notre série de phénotype spécifique épididymo-vésiculo-déférentiel pour les différentes mutations les plus fréquemment recherchées (11 phénotypes différents avec 13 mutations recherchées pour 32 patients dans notre série).

Il semble que l'on puisse rapprocher de l'ABCD, sur le plan de la pathogénie, les AUCD sans anomalie rénale associée.

A l'inverse, l'association de l'absence bi- ou unilatérale des canaux déférents à une agénésie rénale unilatérale (car la forme bilatérale n'est pas viable) mérite le terme d'agénésie déférentielle. Elle correspond à une anomalie de développement du tractus wolffien, dont la forme bilatérale est asymétrique. Ces anomalies partagent le même déterminisme que les kystes congénitaux des vésicules séminales ou des canaux éjaculateurs.

Là encore la vésicule séminale apparaît comme un bon marqueur de l'absence du canal déférent par son absence ou ses anomalies morphologiques.

\section{Les vésicules séminales}

La dilatation des VS est facile à affirmer lorsqu'il existe un kyste de la vésicule séminale, une hypertrophie manifeste avec déshabitation glandulaire ou lorsque la dilatation est unilatérale. Elle est plus difficile à affirmer lorsque les vésicules séminales sont symétriques d'autant que la dilatation est décrite avec le vieillissement, la rareté des rapports sexuels ou les spermatocystites. Une épaisseur $\geq 15 \mathrm{~mm}$ pour affirmer la distension est le cri- 
tère généralement admis. L'IRM montrerait dans les cas d'interprétation difficile un aspect "dépeletonné " [3].

La dilatation des VS incite à rechercher un obstacle d'aval, situé souvent au niveau des canaux éjaculateurs : endoluminal (lithiase) ou par compression en rapport avec un kyste de la base prostatique ou des lésions de prostatite au contact des canaux éjaculateurs (fig.10). Elle peut cependant s'observer sans obstacle, vraisemblablement en rapport avec une atonie, comme dans les polykystoses rénales de l'adulte [6] (fig.11).

La présence de spermatozoïdes à la ponction transrectale échoguidée des VS apparaît comme le témoignage d'un obstacle d'aval [8]. Elle permet pour certains auteurs la réalisation de clichés radiologiques de la voie séminale par injection de produit de contraste $[10,18]$.

\section{Les kystes de la base prostatique}

Les kystes médians sont représentés par les kystes müllériens généralement volumineux, dépassant la base prostatique, responsables d'une compression bilatérale des canaux éjaculateurs (fig.12 et 13) et les kystes de l'utricule prostatique, de plus petite taille (fig.14) $[3,13]$. Ils ne contiennent pas de spermatozoïdes à l'inverse des anomalies d'origine wolffienne sans obstacle d'amont. Ceux-ci correspondent aux kystes congénitaux des vésicules séminales et des canaux éjaculateurs, latéralisés à leur origine, mais pouvant par leur volume retentir sur la voie séminale controlatérale [11]. Ils s'accompagnent généralement, dans leur forme congénitale, d'une agénésie rénale unilatérale avec parfois présence d'un moignon urétéral ectopique dilaté dont seule la terminaison est visible par voie endorectale et dont l'étude bénéficie de séquences d'IRM (fig.15).

Les coupes d'IRM permettent aussi d'obtenir une cartographie précise, en particulier de l'épaisseur prostatique sagittale avant une éventuelle résection transurétrale (RTU), dont le but est de restaurer la perméabilité de la distalité de la voie séminale en effondrant le kyste ou en réséquant l'obstacle situé à la terminaison des canaux éjaculateurs [5,14,22]. L'indication d'une RTU peut parfois se poser, en dehors de l'hypofertilité, devant la persistance de phénoménes infectieux vésiculo-prostatiques récidivants, entretenus par la stase et s'accompagnant volontiers d'hémospermie.

\section{Les infections distales}

Les prostatites aigues et subaigues se traduisent par une plage hypoéchogène arrondie ou cunéiforme plutôt périphérique, avec en phase aiguë une augmentation de volume des plexus veineux périprostatiques et avec le temps un aplatissement du contour en regard d'une plage devenant généralement hyperéchogène (fig.16). Le passage à la chronicité est traduit par l'alternance de plages hyper- et hypoéchogènes avec parfois des calcifications, typiquement de la zone périphérique. Ces lésions lorsqu'elles se produisent au contact des canaux éjaculateurs sont responsables de sténoses visibles par la distension d'amont (fig.17). Mais il n'est pas toujours facile d'affirmer la relation de causalité entre les remaniements, surtout s'ils sont modérés, de l'échostructure au contact des canaux éjaculateurs et les altérations du spermogramme, d'autant que les remaniements hyperéchogènes sont fréquemment retrouvés dans la population normale $[2,9,16]$. A l'inverse, il est probable que certaines obstructions distales des canaux éjaculateurs dont le contenu peut être sensiblement isoéchogène au parenchyme de voisinage échappent au diagnostic qui n'est fondé, lors des explorations en coupes, que sur la distension liquidienne d'amont.

L’infection aiguë des vésicules séminales se traduit par une hypertrophie et il peut exister comme pour la prostate une abcèdation. Au stade chronique, il existe un épaississement des parois externes et des parois glandulaires internes aboutissant à une atrophie (fig.18). La réduction de la lumière des logettes glandulaires est bien visible en IRM. L'IRM permet en effet une analyse fine du contenu des vésicules séminales, très limitée en échographie. En particulier, la présence d'un contenu hémorragique et/ou infecté se traduit par un hypersignal en T1 et un hyposignal en T2, à l'inverse de la normalité. Cet aspect coexiste fréquemment avec un obstacle d'aval (fig.19). Des études par EER ont d'ailleurs montré une cor- 


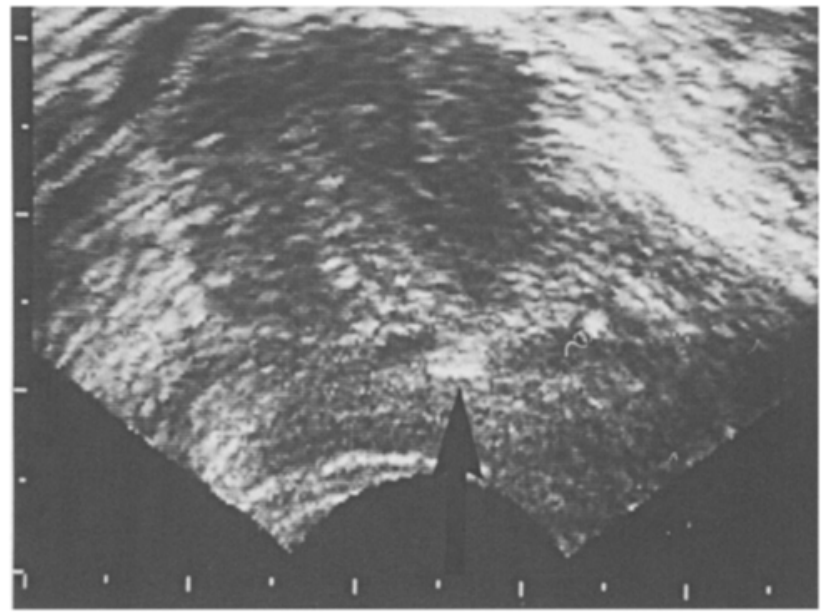

Figure 10 : échographie transrectale, coupe sagittale. Lithiases $(\rightarrow)$ dans un canal éjaculateur dilaté.

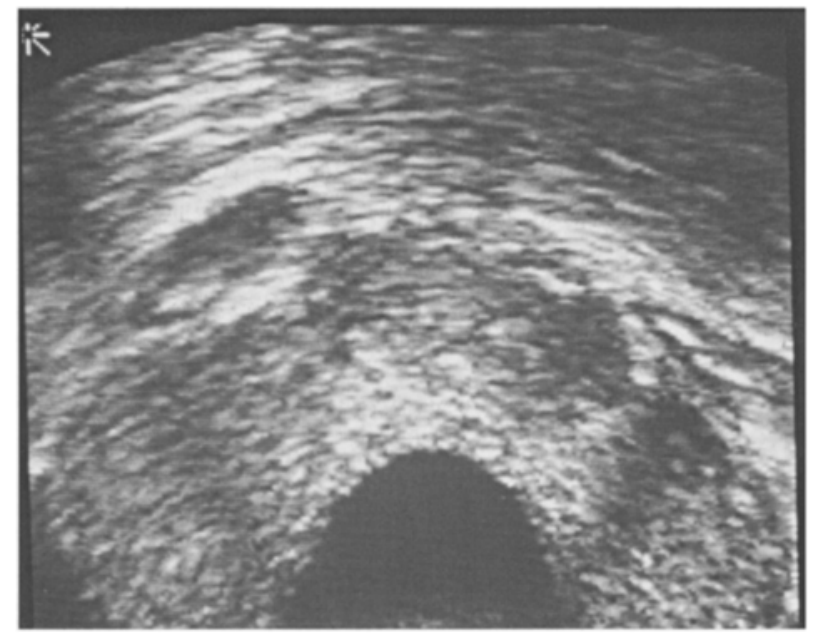

Figure 12 : kyste mullérien. Echographie transrectale, coupe axiale.La partie postérieure et médiane de la base prostatique est occupée par un kyste mullérien, liquidien pur.

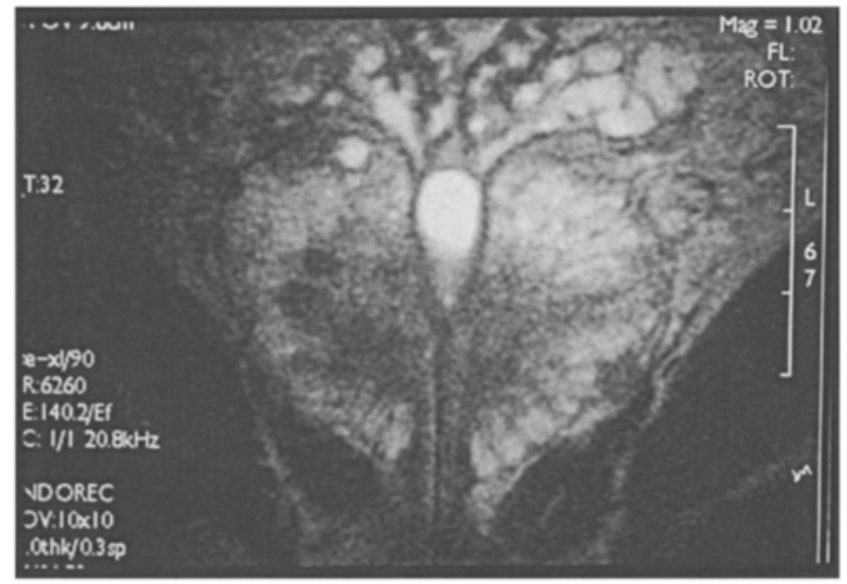

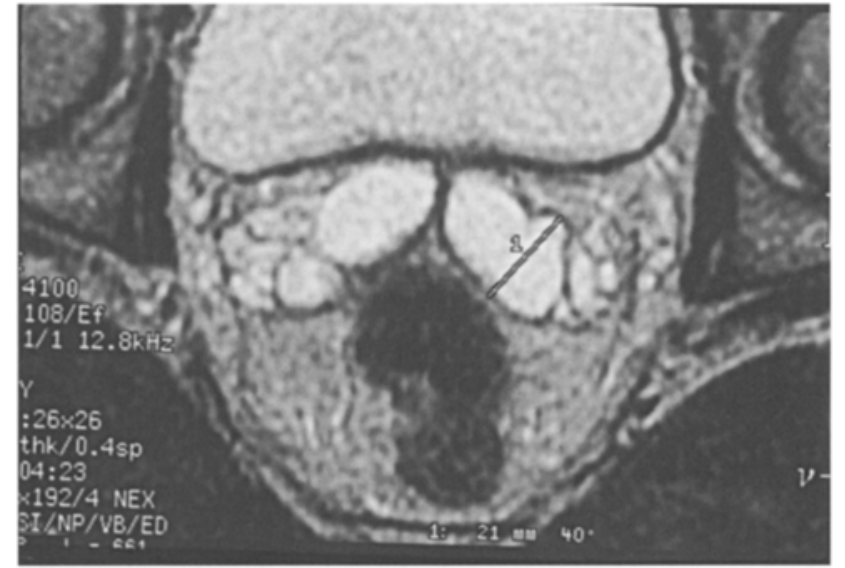

Figure 11 : maladie polykystique rénale. Dilatation des vésicules séminales. IRM, antenne corps, coupe axiale T2. Les VS sont distendues (plus de $20 \mathrm{~mm}$ de diamètre antéro-postérieur) avec une perte de la structure glandulaire surtout dans leur partie interne.

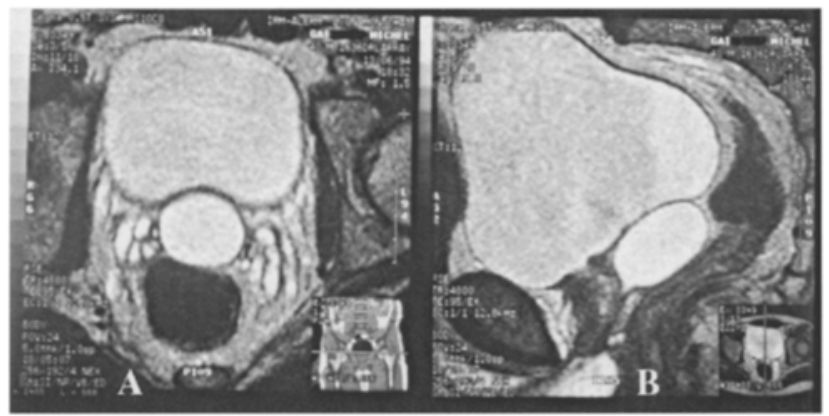

Figure 13 : kyste mullérien. IRM, antenne corps, a) coupe axiale $\mathrm{T2}$ b) coupe sagittale T2. Le volumineux kyste déborde la base prostatique et s'accompagne d'une dilatation des VS par compression des canaux éjaculateurs.

Figure 14 : kyste de l'utricule. IRM avec antenne endorectale. Coupe frontale T2. Le kyste, médian, de petite taille, est responsable d'une compression de la terminaison de la voie séminale avec dilatation des canaux éjaculateurs et des VS qui sont dépelotonnées. 


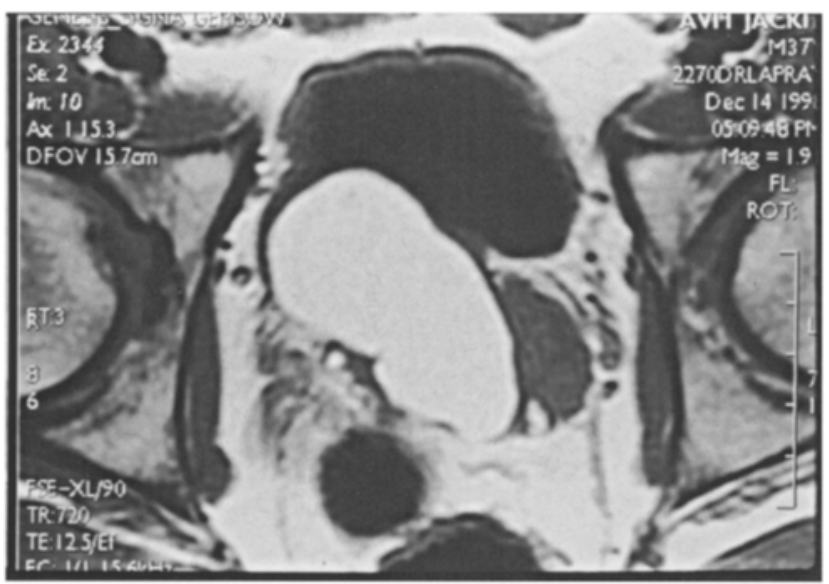

Figure 15 : kyste congénital de la vésicule séminale droite. IRM coupe axiale T1. Le kyste d'environ $6 x 3 \mathrm{~cm}$, en hypersignal témoignant d'un contenu impur, fait protrusion dans la face postérieure de la vessie.

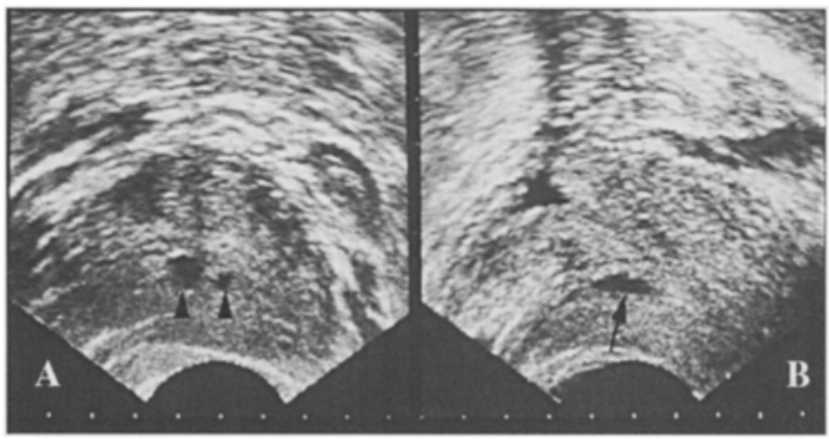

Figure 17 : échographie transrectale en coupe axiale (a) et sagittale (b) montrant la dilatation des canaux éjaculateurs $(\rightarrow)$ au sein d'une prostate hétérogène et hyperéchogène traduisant des lésions de prostatite.

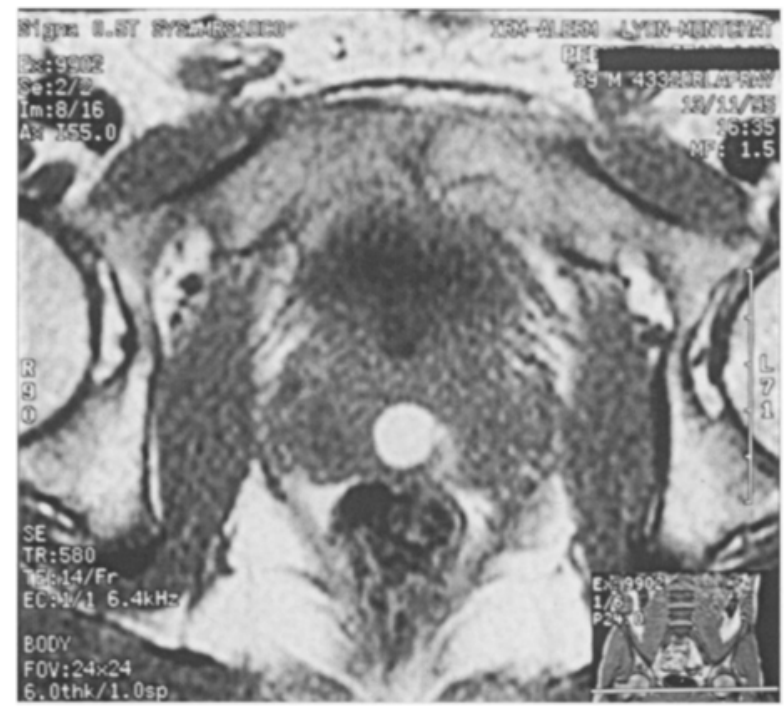

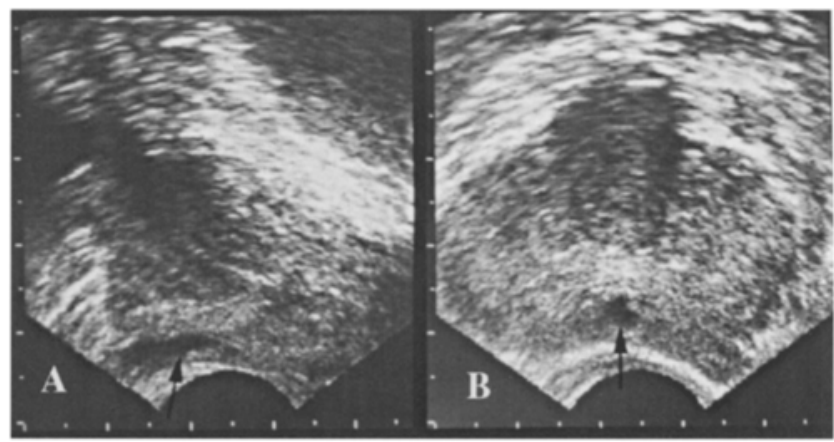

Figure 16 : échographie transrectale en coupe sagittale (a) et axiale (b). Dilatation d'un canal éjaculateur $(\rightarrow)$ au contact de remaniements hyperéchogènes.

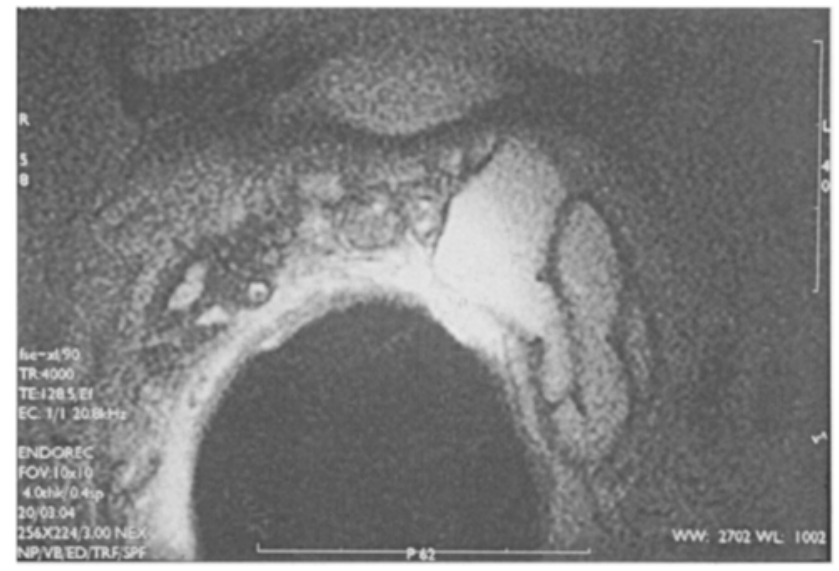

Figure 18 : IRM coupe axiale T2 avec antenne endorectale. Atrophie de la vésicule séminale droite avec épaississement diffus des parois et réduction des lumières glandulaires dans les séquelles d'une inflammation chronique.

Figure 19 : hémospermie récidivante. (a) IRM coupe axiale T2. Les deux VS sont distendues avec présence de sang dans la VS gauche (hypersignal en T1 et atténuation de l'hypersignal en T2) en rapport avec un kyste médian de la base prostatique. 


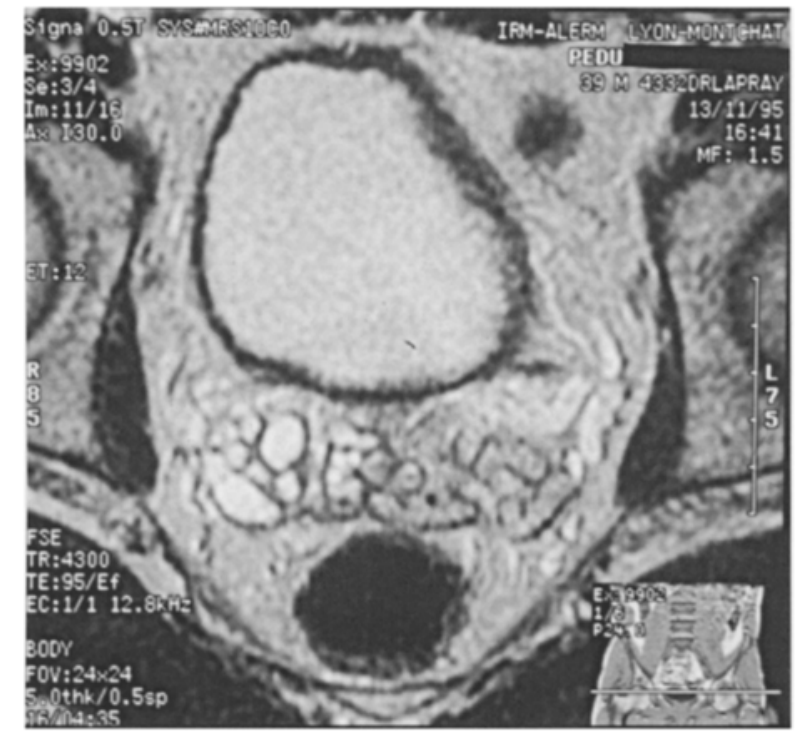

Figure 19 : hémospermie récidivante. (b) IRM coupe axiale $\mathrm{T1}$ montrant le kyste hémorragique. La résection transurétrale amènera la cédation de tous les symptômes.

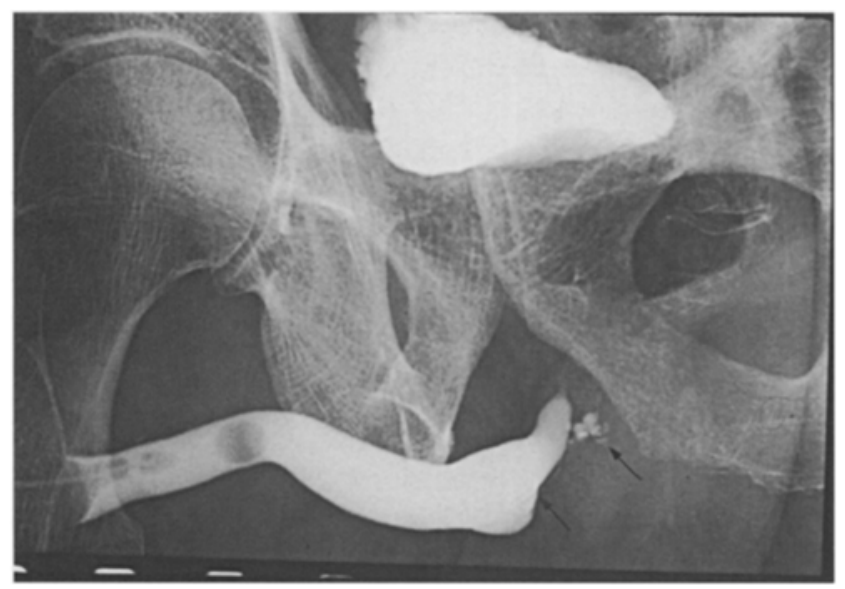

Figure 20 : urétrographie rétrograde. Opacification d'une glande de Cowper dilatée $(\rightarrow)$.

rélation statistique significative entre les calculs des vésicules séminales et l'hémospermie, les calculs des canaux éjaculateurs et une hématurie inexpliquée [12]. Cette pathologie peut d'ailleurs se rencontrer en dehors de l'hypofertilité, dans des syndromes souvent mal étiquetés d'algies pelvi-périnéales.

Les lésions des glandes de Cowper sont rares (fig.20). Les lésions kystiques généralement post-infectieuses, bénéficient de l'exploration en coupes (échographie avec de haute fréquence, IRM) [15].

\section{RÉFÉRENCES}

1. BROWN D.L., BENSON C.B., DOHERTY F.J., DOUBILET P.M., DISALVO D.N. et al. : Cystic testicular mass caused by dilated rete testis. AJR, 1992, 158, 1257-1259.

2. CHRISTIANSEN E., PURVIS K. : Diagnosis of chronic abacterial prostato-vesiculitis by rectal ultrasonography in relation to symptoms and findings. $\mathrm{Br}$. J. Urol., 1991, 67, 173-176.

3. CORNUD F. : L'infertilité masculine. In Ardaens Y., Cornud F. eds. Imagerie et infertilité du couple. Paris, Masson, $1998: 3-57$.

4. GRENIER N., DOUWS C., TRILLAUD H., SAINTAMON A., PAVY S. : Doppler couleur du scrotum. J.E.M.U., 1999, 20, 199-207.

5. HENDRY W.F., PRYOR J.P. : Müllerian duct (prostatic utricle) cyst : diagnosis and treatment in subfertile males. Br. J. Urol.,1992, 69, 79-82.

6. HENDRY W.F., RICKARDS D., PRYOR J.P., BAKER L.R.I. : Seminal megavesicles with adult polycystic kydney disease. Hum. Reprod., 1998, 13 : 1567-1569.

7. HERNANDEZ A.D., URRY R.L., SMITH J.A. : Ultrasonographic characteristics of the seminal vesicles after ejaculation. J. Urol., 1990, 144, 13801382.

8. JAROW J.P. : Seminal vesicle aspiration in the management of patients with ejaculatory duct obstruction. J. Urol.,1994, $152: 899-901$

9. JAROW J.P. : Transrectal ultrasonography of infertile men. Fertil. Steril., 1993, 60 : 1035-1039.

10. JONES T.R., ZAGORIA R.J., JAROW J.P. : Transrectal US-guided seminal vesiculography. Radiology, 1997, 205, 276-278.

11. KING B.F., HATTERY R.R., LIEBER M.M., BERQUIST T.H., WILLIAMSON B., HARTMAN G.W. : Congenital cystic disease of the seminal vesicle. Radiology, 1991, 178, 207-211.

12. LITTRUP P.J., LEE F., MC LEARY R.D., WU D., LEE A., KUMASAKA G.H. : Transrectal US of the seminal vesicles and ejaculatory ducts : clinical correlation. Radiology, 1988, 168 : 625-628.

13. MCDERMOTT V.G., MEAKEM III T.J., STOLPEN A.H., SCHNALL M.D. : Prostatic and periprostatic cysts : findings on MR imaging. AJR, 1995, 164, 123127.

14. MEACHAM R.D., TOWNSEND R.R., DROSE J.A. : Ejaculatory duct obstruction : diagnosis and treatment with transrectal sonography. AJR, 1995, 165, 1463-1466.

15. PARSONS R.B., FISHER A.M., BAR-CHAMA N., MITTY H.A. : MR imaging in male infertility. Radiographics, 1997, $17: 627-637$.

16. POORE R.E., JAROW J.P. : Distribution of intraprostatic hyperechoic lesions in infertile men. Urology, $1995,45,467-469$. 
17. PUTTEMANS T. : Echographie scrotale et infertilité masculine. J.E.M.U., 1999, 20, 192-194.

18. RIEDENKLAU E., BUCH J.P., JAROW J.P. : Diagnosis of vasal obstruction with seminal vesiculography : an alternative to vasography in selected patients. Fertil. Steril. 1995, 64, 1224-1227

19. ROBERT F. : Les absences de canaux déférents dans l'infertilité masculine. Aspects échographiques, génétiques et pathogéniques à propos de 51 cas. Thèse Médecine, Université Claude-Bernard, Lyon, 1998.

20. ROBERT F., ROLLET J., LAPRAY J.F., BEY-OMAR F., DURIEU I., MOREL Y. : Les absences de canaux déférents dans l'infertilité masculine. Presse Med., 1999, 28, 116-121.

21. SECAF E., NURUDDIN R.N., HRICAK H., MCCLURE R.D., DEMAS B. : MR imaging of the seminal vesicles. AJR, 1991, 156, 989-994.

22. TUREK P.J., MAGANA J.O., LIPSHULTZ L.I. : Semen parameters before and after transurethral surgery for ejaculatory duct obstruction. J. Urol., 1996, 155, 1291-1293.

23. VILLERS A., TERRIS M.K., MCNEAL J.E., STAMEY T.A. : Ultrasound anatomy of the prostate : the normal gland and anatomical variations. J. Urol., 1990, 143, $732-738$.

ABSTRACT
Imaging of extratesticular glands of the
seminal tract

LAPRAY J.F., ROLLET J., ROBERT J.

Ultrasonography (US) is presently routine part of the investigation of infertile men with low volume ejaculate. It provides excellent depiction of the congenital or acquired obstructive lesions of the reproductive system, particulary in its distal part. For 10 years, we performed a US examination of the kidneys, the scrotum contents and transrectal US (TRUS) in every case a excretory cause of male hypofertility could be suspected. In selected patients, MR imaging (with endorectal coil) appears usefull.

We describe the normal and abnormal anatomy of the epididymis, vas deferens, seminal vesicle (SV) and ejaculatory ducts in male infertility with US and MRI.

Dilatation of the small ducts in the epididymis and sometimes in the testis at scrotal US is suggestive of a downstream obstacle. Sometimes these dilatation could appear as small hypoechoic areas in the epididymis. In contrast, post-inflammatory changes are hyperechoic but often associated with dilatations.

TRUS permits to confirm the absence of the vas deferens in congenital bilateral absence of vas deferens (CBAVD) : it shows the absence of the ampullae and severe abnormalities of the SV. In case of unilateral absence of the vas deferens, the association with a homolateral kidney agenesis does not lead to the screening for mutations in the cystic fibrosis gene, but suggests a wolffian duct abnormality. MRI (with endorectal coil) is indicated when TRUS is unconclusive.

Scrotal US permits to guide semen aspiration and TRUS is indicated to eliminate a distal obstruction when a surgical anastomosis is planed.

TRUS (as well as scrotal US) can suggest an obstacle when dilatation of one or several segments of the seminal tract is observed. Sometimes the cause is obvious with imaging : CBAVD, prostatic cyst, inflammatory and post-infectious changes, lithiasis etc. However, it remains many cases where US is only one component of the therapeutic decision, besides clinical examination, sperm count, FSH level and biochemical sperm markers.

Key words : male infertility, imaging, ultrasonography, MRI. 